\title{
Role of Main RNA Methylation in Hepatocellular Carcinoma: N6-Methyladenosine, 5-Methylcytosine, and N1-Methyladenosine
}

\author{
Yating $\mathrm{Xu} \mathrm{u}^{1,2,3,4 \dagger}$, Menggang Zhang ${ }^{1,2,3,4 \dagger}$, Qiyao Zhang ${ }^{1,2,3,4 \dagger}$, Xiao $\mathrm{Yu}^{1,2,3,4}$, Zongzong Sun ${ }^{5}$ \\ Yuting $\mathrm{He}^{1,2,3,4 *}$ and Wenzhi Guo ${ }^{1,2,3,4 *}$ \\ ${ }^{1}$ Department of Hepatobiliary and Pancreatic Surgery, The First Affiliated Hospital of Zhengzhou University, Zhengzhou, China, \\ ${ }^{2}$ Key Laboratory of Hepatobiliary and Pancreatic Surgery and Digestive Organ Transplantation of Henan Province, The First \\ Affiliated Hospital of Zhengzhou University, Zhengzhou, China, ${ }^{3}$ Open and Key Laboratory of Hepatobiliary and Pancreatic \\ Surgery and Digestive Organ Transplantation at Henan Universities, Zhengzhou, China, ${ }^{4}$ Henan Key Laboratory of Digestive \\ Organ Transplantation, Zhengzhou, China, ${ }^{5}$ Department of Obstetrics and Gynaecology, The Third Affiliated Hospital of \\ Zhengzhou University, Zhengzhou, China
}

OPEN ACCESS

Edited by:

Ritu Kulshreshtha,

Indian Institute of Technology Delhi,

India

Reviewed by:

Valeria Specchia,

University of Salento, Italy

Lin Zhong,

Shanghai General Hospital, China

Junjun Jia,

Zhejiang Chinese Medical University,

China

${ }^{*}$ Correspondence:

Yuting $\mathrm{He}$

fccheyt1@zzu.edu.cn

Wenzhi Guo

fccguowz@zzu.edu.cn

${ }^{\dagger}$ These authors have contributed equally to this work

Specialty section:

This article was submitted to Epigenomics and Epigenetics,

a section of the journal

Frontiers in Cell and Developmental

Biology

Received: 31 August 2021 Accepted: 18 November 2021 Published: 30 November 2021

Citation:

Xu Y, Zhang M, Zhang Q, YuX, Sun Z, He $Y$ and Guo W (2021) Role of Main

RNA Methylation in Hepatocellular

Carcinoma: N6-Methyladenosine, 5-

Methylcytosine, and N1-

Methyladenosine.

Front. Cell Dev. Biol. 9:767668.

doi: 10.3389/fcell.2021.767668
RNA methylation is considered a significant epigenetic modification, a process that does not alter gene sequence but may play a necessary role in multiple biological processes, such as gene expression, genome editing, and cellular differentiation. With advances in RNA detection, various forms of RNA methylation can be found, including N6methyladenosine (m6A), N1-methyladenosine (m1A), and 5-methylcytosine (m5C). Emerging reports confirm that dysregulation of RNA methylation gives rise to a variety of human diseases, particularly hepatocellular carcinoma. We will summarize essential regulators of RNA methylation and biological functions of these modifications in coding and noncoding RNAs. In conclusion, we highlight complex molecular mechanisms of m6A, $\mathrm{m} 5 \mathrm{C}$, and $\mathrm{m} 1 \mathrm{~A}$ associated with hepatocellular carcinoma and hope this review might provide therapeutic potent of RNA methylation to clinical research.

Keywords: hepatocellular carcinoma, RNA methylation, M6A, m5C, m1A

\section{INTRODUCTION}

Hepatocellular carcinoma (HCC) is a common global disease. It has a poor prognosis and has become the third cause of cancer death (Couri and Pillai, 2019; Hua et al., 2019; Huang et al., 2021). Although medical technology has significantly improved in recent years, 5-years survival rates of patients remain low (8.5\%) (Cai et al., 2019; Zhou et al., 2020). Mortality due to HCC remains high for several reasons. On one hand, clinical symptoms in the early stage are generally displayed uncharacteristic and the lack of effective diagnostic biomarkers, so many patients are easily misdiagnosed. On the other hand, as a result of affluent blood supply in the liver, tumor cells frequently proliferate at a growing rate and distant metastasis tends to appear in the early stage of cancer. Therefore, HCC seriously threatens human health and well-being and is seen as a tough challenge in clinical study. Emerging research is exploring the understanding of pathogenesis in HCC to prevent the dilemma of poor prognosis. Previous reports have showed that the leading pathogenic factor is chronic infection with virus, such as hepatitis $B$ virus and hepatitis $C$ virus 
(Koshiol et al., 2021; Teng et al., 2021). Nevertheless, other molecular mechanisms involved in proliferation, invasion, metastasis, and chemoresistance in HCC remain unknown. Consequently, it is crucial to further investigate the complex mechanisms of tumorigenesis and tumor progression to discover novel makers and identify therapeutic targets.

RNA methylation is commonly regarded as posttranscriptional modification with multiple forms (Chen et al., 2019; Kagra et al., 2021). Although epigenetic modification of RNA has been documented over several decades (Cohn, 1960; Dubin and Taylor, 1975; Perry et al., 1975), our understanding of its biological functions is still limited. Recent research demonstrates that RNA modification may impact RNA metabolism, splicing, stability, and translation (Xue et al., 2020; Nombela et al., 2021), which distinctly influence gene expression. Thus, the effect of RNA methylation is gradually attracting broad attention in a broad array of specialties. For example, numerous investigations verified that $\mathrm{m} 5 \mathrm{C}$ methylation in the $3^{\prime}$-UTR of mRNA increases translation efficiency (Schumann et al., 2020). Occurrence of RNA methylation ordinarily requires the participation of a large number of specific proteins called RNA-modifying proteins (RMPs), containing "writers," "erasers," and "readers" (Frye et al., 2018; Patil et al., 2018). "Writers" are a group of enzymes that catalyze methylation. In contrast, "erasers" are able to remove the decorate of methylation in RNA (Torres and Fujimori, 2015; Shi et al., 2019). "Readers" are a variety of proteins that recognize methylation sites catalyzed by "writers" and bind these sites to form complexes to affect the functions of RNA (Pozner et al., 2018; Grimanelli and Ingouff, 2020; Guo et al., 2020).

A prior survey revealed that abnormal regulation of these RMPs would give rise to incidence of various malignant tumors (Pan et al., 2018; Lan Q. et al., 2019; Zhuang et al., 2020; Li Y. et al., 2021). For instance, catalysis of m6A modification is mediated by methyltransferase-like 3 (METTL3), which is expressed at a high level in colon cancer. Previous evidence showed that METTL3 promoted miRNA-1246 upregulation and induced metastasis in colon cancer (Peng et al., 2019). In bladder cancer, YBX1 obviously emerged the appearance of overexpression than normal tissue. YBX1 is an RMP "reader" in $\mathrm{m} 5 \mathrm{~A}$ modification, and might advance the expression of the multidrug resistance-1 (MDR-1) gene to decrease sensitivity to chemotherapy drugs (Yamashita et al., 2017). Additionally, downregulation of DKC1 was found in breast cancer and gave rise to the impairment result of hTR stabilization (Montanaro et al., 2006). Similarly, overexpression of ALKBH3, methylating affluent m1A modification, ordinarily predicts a dismal prognosis in Hodgkin lymphoma (Yin et al., 2020). Other reports showed that PTR can lengthen survival time during stage M1a of nonsmall cell lung carcinoma (Li et al., 2019; Li H. et al., 2020), and was expected to be incorporated into promising therapeutic strategies for diagnosing patients with evolving ipsilateral pleural dissemination. Various experiments were testified that regulators of RNA methylation, like $\mathrm{m} 6 \mathrm{~A}, \mathrm{~m} 5 \mathrm{C}$, and $\mathrm{m} 1 \mathrm{~A}$, participated in essential biological process for diverse cancers (Li C.-L. et al., 2020). However, discussions that effect of these regulatory factors in RNA methylation related to pathogenesis of
HCC, are constricted in clinical study. Accordingly, in this review we illustrate functional consequences of $\mathrm{m} 6 \mathrm{~A}, \mathrm{~m} 5 \mathrm{C}$, and $\mathrm{m} 1 \mathrm{~A}$ in diverse RNAs. Cooperatively, we focus on targeting RMPs for clinical treatment in HCC in anticipation of providing patients with more promising overall survival and brighter futures.

\section{RNA METHYLATION}

Methylation refers to epigenetic transformations to influence gene expression but does not alter gene sequence, which can be mainly found in DNA, RNA, and protein (Wang X. et al., 2020; Hop et al., 2020; Anton and Roberts, 2021). As approaches to detect RNA modifications improve, RNA methylation began to broadly draw public notice. Multiple functional effects of modification of RNAs were further discussed, and the role of RNA methylation correlative with a variety of cancers also gradually become clearer. We briefly generalize these forms of methylating modification as follows.

\section{N6-Methyladenosine}

$\mathrm{m} 6 \mathrm{~A}$ is methylated adenosine at the nitrogen -6 position and was identified as a posttranscriptional modification in 1974 (Desrosiers et al., 1974). Previous survey has considered that m6A modifications are one of the most extensive methods of RNA methylation in mammals. It was estimated that m6A methylation was approximately present on a quarter of mRNAs (Meyer and Jaffrey, 2017; Chen et al., 2018). As approaches of detecting $\mathrm{m} 6 \mathrm{~A}$ is distinctly preferred, the utilization of ultraviolet crosslinking step realized the new outcome, identifying $\mathrm{m} 6 \mathrm{~A}$ positions at single-nucleotide resolution. Specific locations of $\mathrm{m} 6 \mathrm{~A}$ modification are detected, such as $3^{\prime}$ untranslated regions ( $3^{\prime}$-UTRs) (Dominguez et al., 2018), long internal exons, intergenic regions, introns, and 5' UTRs. METTL3 was first identified in the occurrence of m6A modification. METTL3 functions as the regulator mediating the export of mRNA by interacting with Per2 and Arntl. In addition, METTL3 can recruit eukaryotic translation initiation factor eIF3 to directly regulate translation flexibly (Lin et al., 2016; Choe et al., 2018). The other "writers" of m6A include METTL14, Wilms tumor 1-associated protein (WTAP), and RNAbinding motif protein 15 (RBM15) (Liu et al., 2014; Wen et al., 2018). Accordingly, "readers" of m6A are primarily proteins in the YT521-B homology (YTH) domain family and include YTHDF1, YTHDF2, YTHDF3, YTHDC1, and YTHDC2. Accumulating evidence demonstrates that promotion of translation can positively modulate the effect of YTHDF1. YTHDF3 accelerates protein synthesis by binding YTHDF1 to mediate ribosomal proteins (Shi H. et al., 2017; Li et al., 2017). In m6A methylation, fat mass and obesityassociated protein (FTO) and alkB homologue 5 (ALKBH5) are considered "erasers". FTO binds to introns of nascent mRNA molecules to modulate the biological process of splicing in mRNA (Bartosovic et al., 2017). Similarly, multiple reports also confirmed that ALKBH5 is a pivotal factor to participate in mRNA splicing (Zheng et al., 2013). 


\section{5-Methylcytosine}

$\mathrm{m} 5 \mathrm{C}$ is defined as the accession of methyl group on the fifth carbon atom of cytosine (Motorin et al., 2010; Huang et al., 2019). Abundant m5C occurs in a variety of RNAs, including mRNA, tRNA, rRNA, viral RNA, vault RNA, and IncRNA. In humans, m5C is introduced by NSUN family members and DNA methyltransferase 2 (DNMT2). NSUN2 methylates primarily tRNA and mRNA. The defined regions of tRNA are the variable loop and leucine at the wobble position (Hussain et al., 2013; Khoddami and Cairns, 2013). In mRNA, the specific sites of catalysis by NSUN2 are the region near the start codon and the noncoding 3 UTR. Distribution of NSUN2 is unique, because of converting altogether with different alteration of cell division cycle. NSUN2 can be found at the nucleolus in G1 phase, whereas it is located in the region between the nucleolus and nucleoplasm in S phrase. NSUN2 starts to gradually appear in the cytoplasm in G2 and M phase (Motorin et al., 2010). It was reported that centrioles could be detected abundant depositions of NSUN2 during M phrase. Previous study declared that NSUN2 played an indispensable role in phosphorylation, protein synthesis, cell cycle progression, and epidermal differentiation and tumorigenesis. NSUN4 and NSUN5 primarily catalyze methylation modification in $25 \mathrm{~s}$ rRNA. NSUN4 protein is frequently found in mitochondria, but NSUN5 is distributed in the nucleolus. Overexpression of NSUN5 promotes synthesis of survival protein to enhance the response to oxidative stress (Schosserer et al., 2015). Most NSUN1 factors are detected in the nucleolus, although a few are detected in the cytoplasm. NSUN1 was found to participate in malignant invasion, cell cycle progression, and formation of chromatin (Sharma et al., 2013). NSUN3 and DNMT2 methylate tRNA and are distributed in mitochondria and cytoplasm, respectively. Numerous experiments indicated that DNMT2 has a critical influence in tumorigenesis, protein synthesis, cell differentiation, and HIV-1 RNA replication (Dev et al., 2017). The "erasers" of $\mathrm{m} 5 \mathrm{C}$ methylation are primarily TET family members. TET1 catalyzes the removal of methylation in coding and non-coding RNAs. In addition, several reports suggested ALYREF recognizes and binds the methyl group catalyzed by NSUN2 in mRNA. ALYREF and NSUN2 together promote the transport of mRNA and increase the efficiency of nuclear-cytoplasmic shuttling (Shi M. et al., 2017). Upregulated YBX1 was observed in the cytoplasm and exerted a positive effect on mRNA stabilization, embryogenesis, and tumorigenesis (Yang et al., 2019).

\section{N1-Methyladenosine}

$\mathrm{m} 1 \mathrm{~A}$ is methylation at the N1 position of adenosine and is capable of altering RNA secondary structure. A previous study identified $\mathrm{m} 1 \mathrm{~A}$ in tRNA, rRNA, mRNA, and mitochondrial RNA. Affluent m1A modification is observed in tRNA and rRNA, while the level of m1A remains low in mRNA. The occurrence of mlA methylation in mRNA is represents a six-fold reduction compared to that of m6A methylation (Dominissini et al., 2016); however, m1A can be found in the coding sequence (CDS), $5^{\prime}$-UTR (Li et al., 2016), and $3^{\prime}$ UTRof mRNA. Emerging survey suggested method of $\mathrm{m} 1 \mathrm{~A}$ modification involving in protein synthesis, which improved the efficiency of translation by inhibiting binding of the releasing factor. In contrast, when m1A methylation occurs in the region of mRNA CDS, translation is suppressed to some degree. TRMT10C and TRMT61B serve as "writers" to participating in catalyzing m1A at position 9 and 58 of tRNA (Chujo and Suzuki, 2012). ALKBH3 and ALKBH1 not only demethylate the reversible modification of m6A, but are found to remove m1A (Liu F. et al., 2016). ALKBH3 can function as a repair enzyme to restore N-methylated bases. Recent investigations clarified that demethylation by ALKBH3 might improve the efficiency of translation. Therefore, silencing of ALKBH3 may have the effect of impeding protein synthesis by enhancing the level of $\mathrm{m} 1 \mathrm{~A}$ in tRNA. Moreover, ALKBH3 was regarded as prostate cancer antigen-1 (PCA-1) (Shimada et al., 2009; Yamato et al., 2012). Upregulation of ALKBH3 was observed in a variety of cancers, which stimulated angiogenesis and inhibited apoptosis in prostate cancer and pancreatic cancer patients. The present study suggested the function of m1A58 may result in decreased translation initiation. When ALKBH1 demethylates $\mathrm{m} 1 \mathrm{~A}$, the elongation phase of translation might be impacted through reduced tRNA usage in protein synthesis (Haag et al,, 2016; Kawarada et al., 2017).

\section{FUNCTIONAL CONSEQUENCES OF RNA METHYLATION}

RNA methylation takes place in various RNAs, which give rise to different outcomes to influence RNA function (Gilbert et al., 2016). The detailed functional consequences associated with modifications $\mathrm{m} 6 \mathrm{~A}, \mathrm{~m} 5 \mathrm{C}$, and $\mathrm{m} 1 \mathrm{~A}$ in RNAs are presented in Table 1.

\section{Role of m6A in RNA}

The stability of mRNA is mainly regulated by modification $\mathrm{m} 6 \mathrm{~A}$. YTHDF2, an m6A "reader", might recruit mRNA into processing bodies and participate in the process of degradation to stabilize mRNA (Wang et al., 2014; Huang et al., 2018). Numerous studies reported that YTHDC1 is involved in triggering the SRSF3 pathway to mediate dynamic splicing of precursor mRNA (Molinie et al., 2016). Furthermore, YTHDC1 promotes the export of mRNA (Roundtree et al., 2017; Lesbirel et al., 2018), recruits nuclear transport receptors, and interacts with TREX mRNA adducts. In contrast, depletion of ALKBH5 accelerates export of mRNA (Zheng et al., 2013). Nuclear export is indispensable for translation of mRNA to protein. Several reports corroborated that METTL3 and METTL14 catalyze the modification $\mathrm{m} 6 \mathrm{~A}$ in the region of the $3^{\prime}$-UTR in p21 mRNA and positively increase the efficiency of translation. The recruitment of DCGR8 is mediated by METTL14 in pri-miRNA to encode and regulate the level of miR-126a (Wang et al., 2014). METTL14 is also important for transcriptional elongation of chromatin, which brings about the outcome of recruiting the microprocessor complex (Nombela et al., 2021).

\section{Role of m5C in RNA}

In mRNA, modification $\mathrm{m} 5 \mathrm{C}$ can have a significant impact on metabolism. ALYREF was considered to enhance the efficiency of 
TABLE 1 | The modification results of $\mathrm{m} 6 \mathrm{~A}, \mathrm{~m} 5 \mathrm{C}$, and $\mathrm{m} 1 \mathrm{~A}$ methylation in various RNA.

\begin{tabular}{|c|c|c|c|c|}
\hline RNA type & Regulators & Modification type & Functional consequences & PMID \\
\hline mRNA & YTHDF2 & $\mathrm{m} 6 \mathrm{~A}$ & Enhance stability & $29,476,152$ \\
\hline mRNA & YTHDC1 & $\mathrm{m} 6 \mathrm{~A}$ & Promote export & $30,218,090$ \\
\hline mRNA & ALKBH5 & $\mathrm{m} 6 \mathrm{~A}$ & Promote export & $23,177,736$ \\
\hline mRNA & MEITL3 & $\mathrm{m} 6 \mathrm{~A}$ & Elevate translational efficiency & $27,117,702$ \\
\hline mRNA & METTL14 & $m 6 A$ & Elevate translational efficiency & $24,284,625$ \\
\hline mRNA & ALYREF & m5C & Promote export & $28,418,038$ \\
\hline mRNA & NSUN2 & m5C & Promote transport and affect protein synthesis (promote, and inhibit) & $25,063,673$ \\
\hline tRNA & NSUN2 & $\mathrm{m} 5 \mathrm{C}$ & Enhance stability and promote survival proteins synthesis to repose stress & $28,062,751$ \\
\hline rRNA & NSUN5 & $\mathrm{m} 5 \mathrm{C}$ & Enhance stability & $27,167,997$ \\
\hline mRNA & - & m1A & Enhance stability and affect translation efficiency (promote, and inhibit) & $28,230,814$ \\
\hline tRNA & - & $\mathrm{m} 1 \mathrm{~A}$ & Promote HIV replication & $29,908,293$ \\
\hline tRNA & ALKBH1 & $\mathrm{m} 1 \mathrm{~A}$ & Enhance stability & $27,984,735$ \\
\hline
\end{tabular}

nuclear-cytoplasmic export by forming a complex with mRNA. Previous experiments confirmed that NSUN2 meditates mRNA transport, which facilitates ALYREF binding to mRNA (Yang et al., 2017). Therefore, m5C affects protein synthesis to a degree. For instance, modification m5C appears in CDS in mRNA, which impairs translation and reduces its efficiency. On the contrary, when $\mathrm{m} 5 \mathrm{C}$ is located at the $3^{\prime}$-UTR, the productivity of protein synthesis is distinctly improved. Diverse locations of m5C might lead to different functional results. Moreover, the modification m5C might maintain the stability of mRNA and facilitate plant development. NSUN2 might mediate root-development-related transcripts to suppress root decay. The present survey found that NSUN2 and DNMT2 mediate m5C methylation and commonly play an essential role in stabilizing tRNA. When cells were exposed to hydrogen peroxide, NSUN2 generated survival proteins to respond to the stress (Blanco et al., 2014; David et al., 2017). DNMT maintains the stability of tRNA Asp-GTC and tRNA Gly-GCC and increases the efficiency of polypeptide synthesis (Tuorto et al., 2015). Several evidences suggest NSUN5 modulates rRNA stability under conditions of oxidative stress (Schosserer et al., 2016). NSUN4 impacts regulation of the last step of ribosomal biogenesis (Metodiev et al., 2014).

\section{Role of m1A in RNA}

Modification $\mathrm{m} 1 \mathrm{~A}$ is found predominantly in structured regions of the $5^{\prime}$-UTR and near alternative start codons, indicating that $\mathrm{m} 1 \mathrm{~A}$ is significantly involved in stabilizing mRNA structure. The accomplishment of m1A methylation also exerts an indispensable effect on translation efficiency. For instance, m1A in the CDS region of mRNA has been considered to block the productivity of protein synthesis because it disrupts Watson-Crick base pairing. The presence of $\mathrm{m} 1 \mathrm{~A}$ might be vital to regulate the structural thermostability of tRNAs. It was reported that m1A together with other post-transcriptional modifications is capable of enhancing the melting temperature of tRNAs. ALKBH1 deficiency improves the cellular level of tRNA-Met to maintain the functional effect of m1A, stabilizing tRNA-Met (Liu F. et al., 2016). On the contrary, deficiency of enzymes catalyzing the achievement of m1 A, have the possibility of induce thermosensitivity (Oerum et al., 2017). Moreover, m1A in tRNA-Lys was found to play an important role in reverse transcription fidelity and participate in the process of HIV replication.

\section{MECHANISM OF RNA METHYLATION IN HCC}

Recently emerging evidence has demonstrated that RNA methylation plays a dramatic role in tumorigenesis, invasion, and migration of HCC and elucidated complex mechanisms. We present the evidence for regulators and the effect of $\mathrm{m} 6 \mathrm{~A}, \mathrm{~m} 5 \mathrm{C}$, and $\mathrm{m} 1 \mathrm{~A}$ related to initiation and progression of HCC in Table 2.

\section{m6A Links to HCC}

Numerous studies have recently probed the relationships between m6A methylation and HCC pathogenesis. Wang et al. demonstrated that circ-KIAA1429 is expressed at a higher level in HCC cells than in normal cells, and the patients generally have shorter survival times (Wang M. et al., 2020). In addition, upregulated circ-KIAA1429 can be found in node metastasis status. These results indicate the fact is that KIAA1429 serves as an oncogene to further HCC invasion and migration by altering the methylation of m6A in ID2 and GATA3 mRNA (Lan T. et al., 2019; Cheng et al., 2019). Previous evidence revealed that Zeb1 was considered to be the downstream target of KIAA1429. Meanwhile, YTHDF3 is able to increase the stability of Zeb1 mRNA, which participates in HCC tumorigenesis. The lifetime of Zeb1 gain improved via the effect of m6A modification (Wang M. et al., 2020). It was reported that circ-KIAA1429 contributed to the growing of invasion and metastasis process in HCC together with the mechanism of m6A-YTHDF3-Zeb1. Chen et al. demonstrated that elevated expression of ALKBH5 can be seen as a critical suppressor to impede proliferation and invasion of HCC by regulating the downstream target LYPD1. In HCC, LYPD1 is considered the oncogene that triggers the physiological process. Silencing of LYPD1 impairs growth and invasion of HCC. ALKBH5 is capable of modulating m6A modification and is involved in the IGF2BP1-associated pattern to regulate target LYPD1 (Chen et al., 2020).

Previous survey unraveled HBXIP and METTL3 maintained high level in HCC patients. HBXIP could stimulate the occurrence of HCC cell malignant behaviors through the upregulation of METTL3 (Yang et al., 2021), catalyzing m6A methylation. METTL3 boosts HCC progression via posttranscriptional silencing of SOCS2 (Chen et al., 2018), whereas METTL3 knockdown reversed these effects by reducing m6A 
TABLE 2 | The association of m6A, m5C, and m1A methylation in HCC.

\begin{tabular}{|c|c|c|c|c|c|c|}
\hline $\begin{array}{l}\text { Modification } \\
\text { type }\end{array}$ & Regulators & Expression & Clinical characters & $\begin{array}{l}\text { Function in } \\
\text { HCC }\end{array}$ & Target & PMID \\
\hline $\mathrm{m} 6 \mathrm{~A}$ & ALKBH5 & Down & Favorable prognosis & Inhibit proliferation and invasion & LYPD1 & $32,772,918$ \\
\hline $\mathrm{m} 6 \mathrm{~A}$ & METTL3 & Up & Poor prognosis & Promote vascular invasion, and metastasis & HBXIP & $33,305,825$ \\
\hline $\mathrm{m} 6 \mathrm{~A}$ & YTHDF3 & Up & Poor prognosis & promote invasion, migration, and EMT & Zeb1 & $32,653,519$ \\
\hline m6A & METTL14 & Down & Favorable prognosis & Inhibit invasion, migration, and EMT & $\begin{array}{l}\text { EGFR/ } \\
\text { PI3K/Akt }\end{array}$ & $33,380,825$ \\
\hline m6A & YTHDF1 & Up & Poor prognosis & Promote proliferation, migration, and invasion & $\begin{array}{l}\text { PI3K/Akt/ } \\
\text { mTOR }\end{array}$ & $34,088,349$ \\
\hline m6A & FTO & Up & Poor prognosis & $\begin{array}{l}\text { Promote initiation, metastasis, and } \\
\text { chemoresistance }\end{array}$ & AMD1 & $33,783,988$ \\
\hline m5C & NSUN2 & Up & $\begin{array}{l}\text { Poor prognosis and advanced TNM } \\
\text { stage }\end{array}$ & Promote metastasis & $\mathrm{H} 19$ & $32,978,516$ \\
\hline m5C & NSUN4 & Up & Poor prognosis & - & - & $32,269,723$ \\
\hline m5C & ALYREF & Up & Poor prognosis & - & - & $32,944,246$ \\
\hline $\mathrm{m} 1 \mathrm{~A}$ & TRMT6 & Up & Poor prognosis & - & PI3K/Akt & $32,934,298$ \\
\hline
\end{tabular}

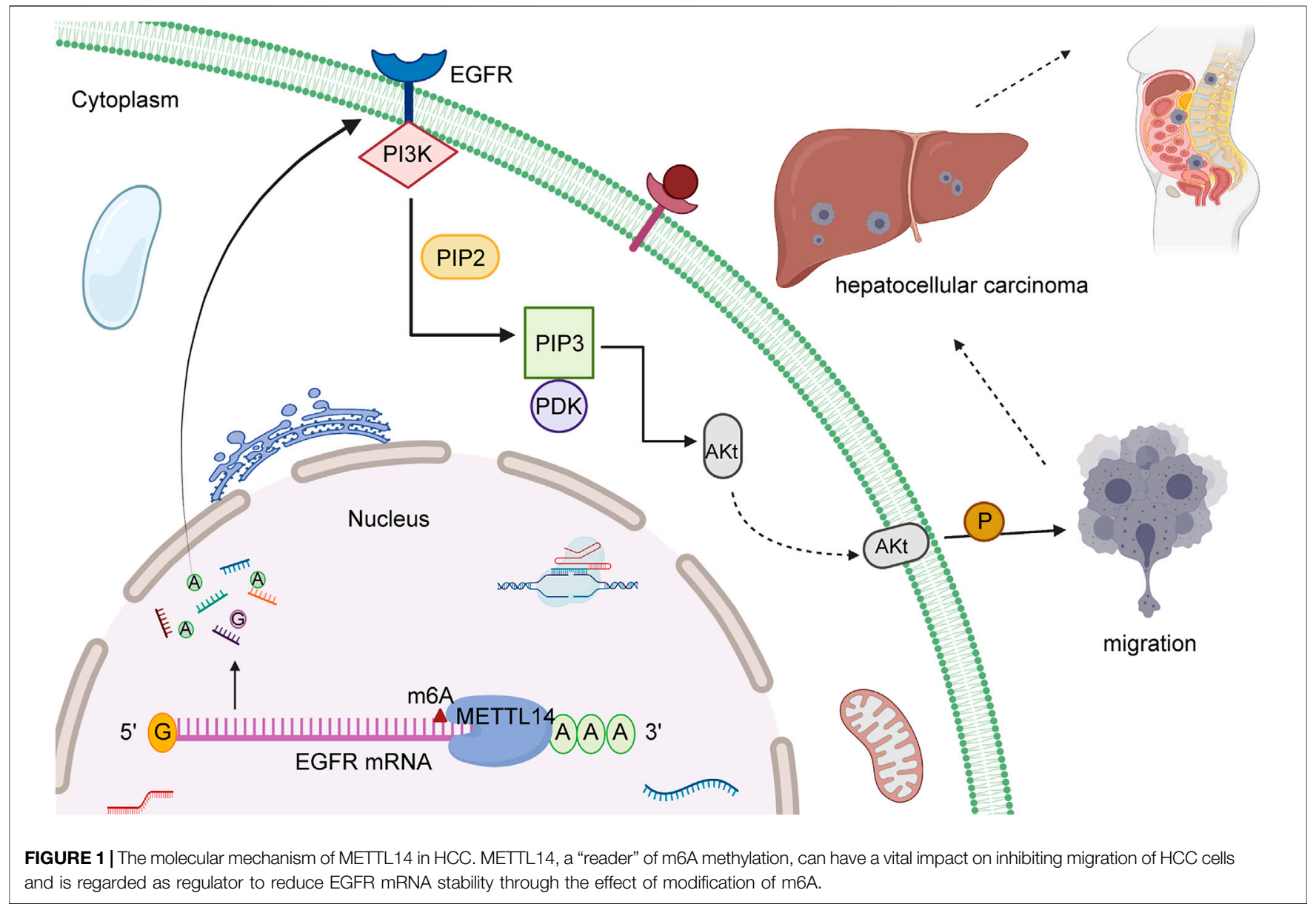

methylation. In contrast, METTL14 has been found to block the metastasis program of HCC, which decreases the stability of EGFR mRNA via posttranscriptional modification of $\mathrm{m} 6 \mathrm{~A}$ in Figure 1. EGFR was reported to play a critical role in the pathogenesis of various malignant tumors, such as in breast, pancreatic, prostate, colorectal, and liver cancer. In HCC, EGFR has been confirmed to stimulate the PI3K-AKT signaling pathway and foster the invasive and metastatic capacity of cells. These evidences suggested that EGFR might have the potential to become the promising target for treatment of 


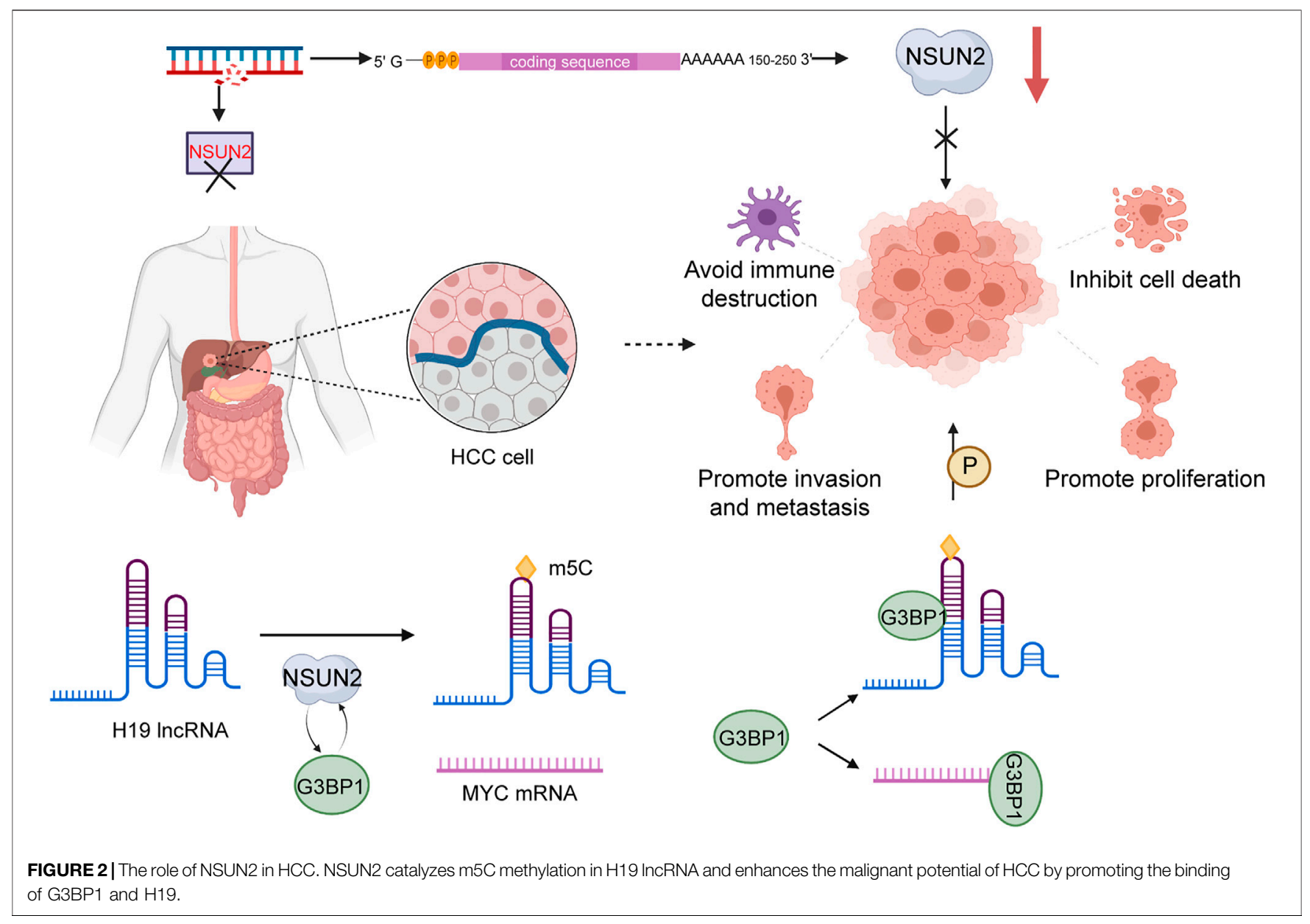

HCC. Consequently, upregulated METTL14 effectively prevents migration of HCC cells, and is associated with positive prognostic outcome in a majority of patients. The suppressive property of METTL14 was revealed in a number of experiments (Shi Y. et al., 2020). Li et al. displayed that YTHDF1, a "reader" of m6A methylation, is upregulated in patients related to HCC and ordinarily is associated with dismal prognosis. HIF-1a interacts with YTHDF1 promoters, and upregulation of YTHDF1 was observed in a HIF-1a dependent manner. HIF$1 \alpha$ has been widely identified to trigger the transcriptional target gene to respond to hypoxic stress. HIF-1a avoids enzymatic degradation during hypoxic stress (Li Q. et al., 2021). YTHDF1 expression, mediated by HIF-1a, supports that hypoxic stress might lead to the alteration of cancer epigenetics, such as the translation of m6A-modified oncogenic mRNAs, to facilitate HCC malignancy. A recent study corroborated that AMD1 expression is the independent factor for overall survival (OS) and disease-free survival (DFS) in HCC (Bian et al., 2021). Several investigations found high expression of AMD1 in HCC tissue and showed that AMD1 regulates the expression of NANOG, SOX2, and KLF4, which are involved in HCC initiation, metastasis, and chemoresistance. Nevertheless, knockdown of AMD1 might increase the sensitivity of HCC cells to sorafenib. Previous report illustrated that FTO can promote the transcription of gene through the effect of removing numerous m6A modifications in the positions of $5^{\prime}$-UTR and CDs. Upregulated FTO could restrain the effect of down-expressed AMD1. While FTO presents the condition of silencing, the effect of AMD1 overexpression will be reversed. As a result, FTO severe as the downstream target of AMD1, and avails the therapeutic advancement for HCC.

\section{m5C Related to HCC}

A recent survey demonstrated that $\mathrm{m} 5 \mathrm{C}$ modification has effects on distribution in HCC tissues and normal tissues. Compared with adjacent non-tumor tissue, high expression of m5C was shown in HCC tissue, which indicated that m5C methylation is closely associated with HCC pathogenesis (He et al., 2020a). NSUN2, a methyltransferase mediating the modification $\mathrm{m} 5 \mathrm{C}$, was confirmed to be upregulated in a variety of tumors in a previous study. Sun et al. showed that NSUN2 is clearly upregulated in HCC tissue have obvious upregulation of NSUN2 than normal tissues, and NSUN2 is capable of promoting the appearance of phenomenon about poor differentiation in HCC. Consistently, NSUN2 knockdown blocked the proliferation, invasion, and migration of HCC cells. Furthermore, NSUN2 have the property of stabilizing H19 by methylating H19 lncRNA. Overexpression of H19 is 
similarly found in HCC tissue with poor prognosis, and H19 is commonly seen as an important feature of poor differentiation in malignancy. Depletion of NSUN2 might give rise to cell inhibition in the G2 phase and prevent the increasing growth of HepG2 cells. Accumulating evidence demonstrates that the distribution of NSUN2 is variable during cell division; expression level is highest in S phase and lowest in G1. These results demonstrate that dynamic expression of NSUN2 has a profound impact on modulating cell division. NSUN2 catalyzed m5C methylation of H19 lncRNA to significantly affect malignant development of HCC. Consequently, H19 has the possibility of becoming a novel target of NSUN2. It was demonstrated that NSUN2 regulates m5C methylation of $\mathrm{H} 19$ lncRNA via interaction of Ras-GTPase-activating protein SH3 domain-binding protein 1 (G3BP1). G3BP1, a known oncoprotein that is generally expressed at a high level in multiple cancers that participate in diverse carcinogenesisassociated pathways containing Ras/MAPK (Liu S.-Y. et al., 2016), Wnt/ $\beta$-catenin, PI3K/AKT (Zhang et al., 2019), and NF- $\kappa \mathrm{B} / \mathrm{Her} 2$ signaling pathways. These pathways could be regulated by NSUN2 through involvement in G3BP1 binding to H19 lncRNA (Sun et al., 2020), playing an essential role in malignant progression of HCC. In addition, G3BP1 also binds MYC mRNA to advance the effect of degradation (Tourrière et al., 2001). H19 IncRNA promotes tumor proliferation by binding G3BP1 and competing with MYC mRNA. When H19 lncRNA is poorly methylated, binding to G3BP1 will be further attenuated. Interestingly, MYC was found to accelerate H19 lncRNA transcription (Barsyte-Lovejoy et al., 2006). Therefore, the MYC-NSUN2-H19-G3BP1 axis was revealed to be associated with malignant behaviors of HCC (Figure 2). Moreover, the methylation modification could bring the decline number of circRNA, resulting in lack of suppression from crucial proteins and inducing the initiation of tumors (He et al., 2020b).

\section{m1A Associated With HCC}

As a burgeoning discussion hotspot, research on $\mathrm{m} 1 \mathrm{~A}$ modification links to multiple cancers is also gradually becoming the basis of extensive concern. The understanding of m1A function related to HCC still requires further exploration. Shi et al. illustrated that TP53 mutations were primarily correlated with regulators mediating $\mathrm{m} 1 \mathrm{~A}$ methylation (Shi Q. et al., 2020). TP53 is a suppressor of various malignancies. However, the occurrence of TP53 mutations rapidly promotes tumorigenesis; for example, TP53 mutations serve as prognostic indicators of short survival time in HCC. Additionally, m1Aassociated regulators expression actively has the impact on promoting progression of high TNM stage, including expression of RMT6, TRMT61A, TRMT10C, and TRMT6. It was reported YTHDF1 is valuable in predicting prognosis due to improving TRMT6 expression. Mounting survey unravel that m1A methylation might be regulated by the PI3K/Akt signaling pathway in HCC. The PI3K/Akt pathway plays a key role in proliferation and inhibition of apoptosis in HCC (Fu et al., 2019;
Zheng et al., 2019). Nevertheless, how the PI3K/Akt pathway is involved in $\mathrm{m} 1 \mathrm{~A}$ and induces the development of HCC still needs further study. These findings suggest $\mathrm{m} 1 \mathrm{~A}$ has the potential to become a valuable biomarker in HCC.

\section{CONCLUSION}

RNA methylation has emerged as the post-transcriptional modification to significantly affect a variety of genes expression processes, which not only has a broad influence on RNA metabolism but alters the function of various RNAs. Numerous proteins regulate methylation, demethylation, and specifically bind to diverse RNAs to promote or inhibit the biological functions, and are referred to, respectively, as "writers," "erasers," and "readers". Prior research found aberrant expression of these regulators might lead to increasing disease. We summarize the distribution and functional consequences of $\mathrm{m} 6 \mathrm{~A}, \mathrm{~m} 5 \mathrm{C}$, and $\mathrm{m} 1 \mathrm{~A}$ modifications to further understand the role of RNA methylation and corresponding physiological mechanisms in HCC. For instance, overexpression of NSUN2 could promote malignant behaviors of HCC. METTL14, the "writer" of m6A, was proved to prevent metastasis of HCC. In this review, we found that RNA methylation may potentially serve as a novel marker and make valuable contributions to diagnosis and treatment in HCC, providing a promising future for a great many patients. Simultaneously, many studies are necessary to further explore and testify for clinical application.

\section{AUTHOR CONTRIBUTIONS}

Xu YT, Zhang MG, and Zhang QY drafted manuscript. Yu X and Sun $\mathrm{ZZ}$ drew the mechanism diagrams. He YT, and Guo WZ conceived of the study and guided the analysis. He YT, and Xu YT edited and reviewed the manuscript. All authors read and approved the final manuscript.

\section{FUNDING}

This work was supported by the National Natural Science Foundation of China (81902832), the Youth Talent Lifting Project of Henan Province (2021HYTP059), Key Scientific Research Project of Henan Higher Education Institutions of China (21A320026), the Leading Talents of Zhongyuan Science and Technology Innovation (214200510027), the Henan Provincial Medical Science and Technology Research Plan (SBGJ2018002), the Science and Technology Innovation Talents in Henan Universities (19HASTIT003), the Outstanding Foreign Scientist Studio in Henan Province (GZS2020004), and the Youth Talent Lifting Project of Henan Province (2021HYTP059). 


\section{REFERENCES}

Anton, B. P., and Roberts, R. J. (2021). Beyond Restriction Modification: Epigenomic Roles of DNA Methylation in Prokaryotes. Annu. Rev. Microbiol. 75, 129-149. doi:10.1146/annurev-micro-040521-035040

Barsyte-Lovejoy, D., Lau, S. K., Boutros, P. C., Khosravi, F., Jurisica, I., Andrulis, I. L., et al. (2006). The C-Myc Oncogene Directly Induces the H19 Noncoding RNA by Allele-specific Binding to Potentiate Tumorigenesis. Cancer Res. 66, 5330-5337. doi:10.1158/0008-5472.Can-06-0037

Bartosovic, M., Molares, H. C., Gregorova, P., Hrossova, D., Kudla, G., and Vanacova, S. (2017). N6-methyladenosine Demethylase FTO Targets PremRNAs and Regulates Alternative Splicing and $3^{\prime}$-end Processing. Nucleic Acids Res. 45, 11356-11370. doi:10.1093/nar/gkx778

Bian, X., Shi, D., Xing, K., Zhou, H., Lu, L., Yu, D., et al. (2021). AMD1 Upregulates Hepatocellular Carcinoma Cells Stemness by FTO Mediated mRNA Demethylation. Clin. Transl. Med. 11, e352. doi:10.1002/ctm2.352

Blanco, S., Dietmann, S., Flores, J. V., Hussain, S., Kutter, C., Humphreys, P., et al. (2014). Aberrant Methylation of T RNA S Links Cellular Stress to Neurodevelopmental Disorders. Embo J 33, 2020-2039. doi:10.15252/ embj.201489282

Cai, K., Li, T., Guo, L., Guo, H., Zhu, W., Yan, L., et al. (2019). Long Non-coding RNA LINC00467 Regulates Hepatocellular Carcinoma Progression by Modulating miR-9-5p/PPARA Expression. Open Biol. 9, 190074. doi:10.1098/rsob.190074

Chen, M., Wei, L., Law, C.-T., Tsang, F. H.-C., Shen, J., Cheng, C. L.-H., et al. (2018). RNA N6-Methyladenosine Methyltransferase-like 3 Promotes Liver Cancer Progression through YTHDF2-dependent Posttranscriptional Silencing of SOCS2. Hepatology 67, 2254-2270. doi:10.1002/hep.29683

Chen, X., Sun, Y.-Z., Liu, H., Zhang, L., Li, J.-Q., and Meng, J. (2019). RNA Methylation and Diseases: Experimental Results, Databases, Web Servers and Computational Models. Brief Bioinform. 20, 896-917. doi:10.1093/bib/bbx142

Chen, Y., Zhao, Y., Chen, J., Peng, C., Zhang, Y., Tong, R., et al. (2020). ALKBH5 Suppresses Malignancy of Hepatocellular Carcinoma via m6A-Guided Epigenetic Inhibition of LYPD1. Mol. Cancer 19, 123. doi:10.1186/s12943020-01239-w

Cheng, X., Li, M., Rao, X., Zhang, W., Li, X., Wang, L., et al. (2019). KIAA1429 Regulates the Migration and Invasion of Hepatocellular Carcinoma by Altering m6A Modification of ID2 mRNA. Onco. Targets Ther. 12, 3421-3428. doi:10.2147/ott.S180954

Choe, J., Lin, S., Zhang, W., Liu, Q., Wang, L., Ramirez-Moya, J., et al. (2018). mRNA Circularization by METTL3-eIF3h Enhances Translation and Promotes Oncogenesis. Nature 561, 556-560. doi:10.1038/s41586-018-0538-8

Chujo, T., and Suzuki, T. (2012). Trmt61B Is a Methyltransferase Responsible for 1-methyladenosine at Position 58 of Human Mitochondrial tRNAs. RNA 18, 2269-2276. doi:10.1261/rna.035600.112

Cohn, W. E. (1960). Pseudouridine, a Carbon-Carbon Linked Ribonucleoside in Ribonucleic Acids: Isolation, Structure, and Chemical Characteristics. J. Biol. Chem. 235, 1488-1498. doi:10.1016/s0021-9258(18)69432-3

Couri, T., and Pillai, A. (2019). Goals and Targets for Personalized Therapy for HCC. Hepatol. Int. 13, 125-137. doi:10.1007/s12072-018-9919-1

David, R., Burgess, A., Parker, B., Li, J., Pulsford, K., Sibbritt, T., et al. (2017). Transcriptome-Wide Mapping of RNA 5-Methylcytosine in Arabidopsis mRNAs and Noncoding RNAs. Plant Cell 29, 445-460. doi:10.1105/ tpc. 16.00751

Desrosiers, R., Friderici, K., and Rottman, F. (1974). Identification of Methylated Nucleosides in Messenger RNA from Novikoff Hepatoma Cells. Proc. Natl. Acad. Sci. 71, 3971-3975. doi:10.1073/pnas.71.10.3971

Dev, R. R., Ganji, R., Singh, S. P., Mahalingam, S., Banerjee, S., and Khosla, S. (2017). Cytosine Methylation by DNMT2 Facilitates Stability and Survival of HIV-1 RNA in the Host Cell during Infection. Biochem. J. 474, 2009-2026. doi:10.1042/bcj20170258

Dominguez, D., Freese, P., Alexis, M. S., Su, A., Hochman, M., Palden, T., et al. (2018). Sequence, Structure, and Context Preferences of Human RNA Binding Proteins. Mol. Cel 70, 854-867. doi:10.1016/j.molcel.2018.05.001

Dominissini, D., Nachtergaele, S., Moshitch-Moshkovitz, S., Peer, E., Kol, N., BenHaim, M. S., et al. (2016). The Dynamic N1-Methyladenosine Methylome in Eukaryotic Messenger RNA. Nature 530, 441-446. doi:10.1038/nature16998
Dubin, D. T., and Taylor, R. H. (1975). The Methylation State of Poly A-Containing-Messenger RNA from Cultured Hamster Cells. Nucl. Acids Res. 2, 1653-1668. doi:10.1093/nar/2.10.1653

Frye, M., Harada, B. T., Behm, M., and He, C. (2018). RNA Modifications Modulate Gene Expression during Development. Science 361, 1346-1349. doi:10.1126/ science.aau1646

Fu, H.-w., Lin, X., Zhu, Y.-x., Lan, X., Kuang, Y., Wang, Y.-z., et al. (2019). CircIGF1R Has Pro-proliferative and Anti-apoptotic Effects in HCC by Activating the PI3K/AKT Pathway. Gene 716, 144031. doi:10.1016/j.gene.2019.144031

Gilbert, W. V., Bell, T. A., and Schaening, C. (2016). Messenger RNA Modifications: Form, Distribution, and Function. Science 352, 1408-1412. doi:10.1126/science.aad8711

Grimanelli, D., and Ingouff, M. (2020). DNA Methylation Readers in Plants. J. Mol. Biol. 432, 1706-1717. doi:10.1016/j.jmb.2019.12.043

Guo, Z., Li, Z., Liu, Y., An, Z., Peng, M., Shen, W. H., et al. (2020). MRG1/2 Histone Methylation Readers and HD2C Histone Deacetylase Associate in Repression of the Florigen Gene FT to Set a Proper Flowering Time in Response to Daylength Changes. New Phytol. 227, 1453-1466. doi:10.1111/nph.16616

Haag, S., Sloan, K. E., Ranjan, N., Warda, A. S., Kretschmer, J., Blessing, C., et al. (2016). NSUN 3 and ABH 1 Modify the Wobble Position of Mt-t RNA Met to Expand Codon Recognition in Mitochondrial Translation. Embo J 35, 2104-2119. doi:10.15252/embj.201694885

He, Y., Shi, Q., Zhang, Y., Yuan, X., and Yu, Z. (2020a). Transcriptome-Wide 5Methylcytosine Functional Profiling of Long Non-Coding RNA in Hepatocellular Carcinoma. Cancer Manag. Res. 12, 6877-6885. doi:10.2147/ cmar.S262450

He, Y., Zhang, Q., Zheng, Q., Yu, X., and Guo, W. (2020b). Distinct 5methylcytosine Profiles of Circular RNA in Human Hepatocellular Carcinoma. Am. J. Transl. Res. 12, 5719-5729.

Hop, P. J., Luijk, R., Daxinger, L., Van Iterson, M., Dekkers, K. F., Jansen, R., et al. (2020). Genome-wide Identification of Genes Regulating DNA Methylation Using Genetic Anchors for Causal Inference. Genome Biol. 21, 220. doi:10.1186/ s13059-020-02114-Z

Hua, X., Sanjiv, K., Gad, H., Pham, T., Gokturk, C., Rasti, A., et al. (2019). Karonudib Is a Promising Anticancer Therapy in Hepatocellular Carcinoma. Ther. Adv. Med. Oncol. 11, 175883591986696. doi:10.1177/ 1758835919866960

Huang, H., Weng, H., Sun, W., Qin, X., Shi, H., Wu, H., et al. (2018). Recognition of RNA N6-Methyladenosine by IGF2BP Proteins Enhances mRNA Stability and Translation. Nat. Cel Biol. 20, 285-295. doi:10.1038/s41556-018-0045-Z

Huang, T., Chen, W., Liu, J., Gu, N., and Zhang, R. (2019). Genome-wide Identification of mRNA 5-methylcytosine in Mammals. Nat. Struct. Mol. Biol. 26, 380-388. doi:10.1038/s41594-019-0218-x

Huang, D. Q., El-Serag, H. B., and Loomba, R. (2021). Global Epidemiology of NAFLD-Related HCC: Trends, Predictions, Risk Factors and Prevention. Nat. Rev. Gastroenterol. Hepatol. 18, 223-238. doi:10.1038/s41575-020-00381-6

Hussain, S., Sajini, A. A., Blanco, S., Dietmann, S., Lombard, P., Sugimoto, Y., et al. (2013). NSun2-mediated Cytosine-5 Methylation of Vault Noncoding RNA Determines its Processing into Regulatory Small RNAs. Cel Rep. 4, 255-261. doi:10.1016/j.celrep.2013.06.029

Kagra, D., Mahmi, A. S., Kumar, N. V. S., Prabhakar, P. S., and Sharma, P. (2021). Influence of the Number, Nature and Position of Methyl Posttranscriptional Modifications on Nucleobase Stacking in RNA. Chemphyschem 22, 1622-1630. doi:10.1002/cphc.202100333

Kawarada, L., Suzuki, T., Ohira, T., Hirata, S., Miyauchi, K., and Suzuki, T. (2017). ALKBH1 Is an RNA Dioxygenase Responsible for Cytoplasmic and Mitochondrial tRNA Modifications. Nucleic Acids Res. 45, 7401-7415. doi:10.1093/nar/gkx354

Khoddami, V., and Cairns, B. R. (2013). Identification of Direct Targets and Modified Bases of RNA Cytosine Methyltransferases. Nat. Biotechnol. 31, 458-464. doi:10.1038/nbt.2566

Koshiol, J., Argirion, I., Liu, Z., Kim Lam, T., O’Brien, T. R., Yu, K., et al. (2021). Immunologic Markers and Risk of Hepatocellular Carcinoma in Hepatitis B Virus- and Hepatitis C Virus-infected Individuals. Aliment. Pharmacol. Ther. 54, 833-842. doi:10.1111/apt.16524

Lan, Q., Liu, P. Y., Haase, J., Bell, J. L., Hüttelmaier, S., and Liu, T. (2019a). The Critical Role of RNA m6A Methylation in Cancer. Cancer Res. 79, 1285-1292. doi:10.1158/0008-5472.Can-18-2965 
Lan, T., Li, H., Zhang, D., Xu, L., Liu, H., Hao, X., et al. (2019b). KIAA1429 Contributes to Liver Cancer Progression through N6-methyladenosinedependent post-transcriptional Modification of GATA3. Mol. Cancer 18, 186. doi:10.1186/s12943-019-1106-Z

Lesbirel, S., Viphakone, N., Parker, M., Parker, J., Heath, C., Sudbery, I., et al. (2018). The m6A-Methylase Complex Recruits TREX and Regulates mRNA export. Sci. Rep. 8, 13827. doi:10.1038/s41598-018-32310-8

Li, X., Xiong, X., Wang, K., Wang, L., Shu, X., Ma, S., et al. (2016). Transcriptomewide Mapping Reveals Reversible and Dynamic N1-Methyladenosine Methylome. Nat. Chem. Biol. 12, 311-316. doi:10.1038/nchembio.2040

Li, A., Chen, Y.-S., Ping, X.-L., Yang, X., Xiao, W., Yang, Y., et al. (2017). Cytoplasmic m6A Reader YTHDF3 Promotes mRNA Translation. Cell Res. 27, 444-447. doi:10.1038/cr.2017.10

Li, H., Sun, Z., Yang, F., Sui, X., Liu, T., and Wang, J. (2019). Primary Tumour Resection in Non-small-cell Lung Cancer Patients with Ipsilateral Pleural Dissemination (M1a): a Population-Based Study. Eur. J. Cardiothorac. Surg. 55, 1121-1129. doi:10.1093/ejcts/ezy439

Li, C.-L., Tang, D.-R., Ji, J., Zang, B., Chen, C., and Zhao, J.-Q. (2020a). Colorectal Adenocarcinoma Patients with M1a Diseases Gain More Clinical Benefits from Palliative Primary Tumor Resection Than Those with M1b Diseases: A Propensity Score Matching Analysis. World J. Clin. Cases 8, 3230-3239. doi:10.12998/wjcc.v8.i15.3230

Li, H., Liu, T., Sun, Z., and Yang, F. (2020b). Primary Tumor Resection of Nonsmall Cell Lung Cancer Patients with Ipsilateral Pleural Dissemination (M1a) in the Era of Targeted Therapy. Thorac. Cancer 11, 3213-3222. doi:10.1111/17597714.13649

Li, Q., Ni, Y., Zhang, L., Jiang, R., Xu, J., Yang, H., et al. (2021a). HIF-1a-induced Expression of m6A Reader YTHDF1 Drives Hypoxia-Induced Autophagy and Malignancy of Hepatocellular Carcinoma by Promoting ATG2A and ATG14 Translation. Sig Transduct. Target. Ther. 6, 76. doi:10.1038/s41392-02000453-8

Li, Y., Qi, D., Zhu, B., and Ye, X. (2021b). Analysis of m6A RNA MethylationRelated Genes in Liver Hepatocellular Carcinoma and Their Correlation with Survival. Int. J. Mol. Sci. 22, 1474. doi:10.3390/ijms22031474

Lin, S., Choe, J., Du, P., Triboulet, R., and Gregory, R. I. (2016). The M 6 A Methyltransferase METTL3 Promotes Translation in Human Cancer Cells. Mol. Cel 62, 335-345. doi:10.1016/j.molcel.2016.03.021

Liu, J., Yue, Y., Han, D., Wang, X., Fu, Y., Zhang, L., et al. (2014). A METTL3METTL14 Complex Mediates Mammalian Nuclear RNA N6-Adenosine Methylation. Nat. Chem. Biol. 10, 93-95. doi:10.1038/nchembio.1432

Liu, F., Clark, W., Luo, G., Wang, X., Fu, Y., Wei, J., et al. (2016a). ALKBH1Mediated tRNA Demethylation Regulates Translation. Cell 167, 1897. doi:10.1016/j.cell.2016.11.045

Liu, S.-Y., Joseph, N. M., Ravindranathan, A., Stohr, B. A., Greenland, N. Y., Vohra, P., et al. (2016b). Genomic Profiling of Malignant Phyllodes Tumors Reveals Aberrations in FGFR1 and PI-3 Kinase/RAS Signaling Pathways and Provides Insights into Intratumoral Heterogeneity. Mod. Pathol. 29, 1012-1027. doi:10.1038/modpathol.2016.97

Metodiev, M. D., Spåhr, H., Loguercio Polosa, P., Meharg, C., Becker, C., Altmueller, J., et al. (2014). NSUN4 Is a Dual Function Mitochondrial Protein Required for Both Methylation of 12S rRNA and Coordination of Mitoribosomal Assembly. Plos Genet. 10, e1004110. doi:10.1371/ journal.pgen.1004110

Meyer, K. D., and Jaffrey, S. R. (2017). Rethinking m6A Readers, Writers, and Erasers. Annu. Rev. Cel Dev. Biol. 33, 319-342. doi:10.1146/annurev-cellbio100616-060758

Molinie, B., Wang, J., Lim, K. S., Hillebrand, R., Lu, Z.-x., Van Wittenberghe, N., et al. (2016). m6A-LAIC-seq Reveals the Census and Complexity of the m6A Epitranscriptome. Nat. Methods 13, 692-698. doi:10.1038/nmeth.3898

Montanaro, L., Brigotti, M., Clohessy, J., Barbieri, S., Ceccarelli, C., Santini, D., et al. (2006). Dyskerin Expression Influences the Level of Ribosomal RNA Pseudouridylation and Telomerase RNA Component in Human Breast Cancer. J. Pathol. 210, 10-18. doi:10.1002/path.2023

Motorin, Y., Lyko, F., and Helm, M. (2010). 5-methylcytosine in RNA: Detection, Enzymatic Formation and Biological Functions. Nucleic Acids Res. 38, 1415-1430. doi:10.1093/nar/gkp1117
Nombela, P., Miguel-López, B., and Blanco, S. (2021). The Role of m6A, m5C and $\Psi$ RNA Modifications in Cancer: Novel Therapeutic Opportunities. Mol. Cancer 20, 18. doi:10.1186/s12943-020-01263-w

Oerum, S., Dégut, C., Barraud, P., and Tisné, C. (2017). m1A Post-Transcriptional Modification in tRNAs. Biomolecules 7, 20. doi:10.3390/biom7010020

Pan, Y., Ma, P., Liu, Y., Li, W., and Shu, Y. (2018). Multiple Functions of m6A RNA Methylation in Cancer. J. Hematol. Oncol. 11, 48. doi:10.1186/s13045-0180590-8

Patil, D. P., Pickering, B. F., and Jaffrey, S. R. (2018). Reading m6A in the Transcriptome: m6A-Binding Proteins. Trends Cel Biol. 28, 113-127. doi:10.1016/j.tcb.2017.10.001

Peng, W., Li, J., Chen, R., Gu, Q., Yang, P., Qian, W., et al. (2019). Upregulated METTL3 Promotes Metastasis of Colorectal Cancer via miR-1246/SPRED2/ MAPK Signaling Pathway. J. Exp. Clin. Cancer Res. 38, 393. doi:10.1186/ s13046-019-1408-4

Perry, R. P., Kelley, D. E., Friderici, K., and Rottman, F. (1975). The Methylated Constituents of L Cell Messenger RNA: Evidence for an Unusual Cluster at the 5' Terminus. Cell 4, 387-394. doi:10.1016/0092-8674(75)90159-2

Pozner, A., Hudson, N. O., Trewhella, J., Terooatea, T. W., Miller, S. A., and BuckKoehntop, B. A. (2018). The C-Terminal Zinc Fingers of ZBTB38 Are Novel Selective Readers of DNA Methylation. J. Mol. Biol. 430, 258-271. doi:10.1016/ j.jmb.2017.12.014

Roundtree, I. A., Luo, G.-Z., Zhang, Z., Wang, X., Zhou, T., Cui, Y., et al. (2017). YTHDC1 Mediates Nuclear export of N6-Methyladenosine Methylated mRNAs. Elife 6, e31311. doi:10.7554/eLife.31311

Schosserer, M., Minois, N., Angerer, T. B., Amring, M., Dellago, H., Harreither, E., et al. (2015). Methylation of Ribosomal RNA by NSUN5 Is a Conserved Mechanism Modulating Organismal Lifespan. Nat. Commun. 6, 6158. doi: $10.1038 /$ ncomms7158

Schosserer, M., Minois, N., Angerer, T. B., Amring, M., Dellago, H., Harreither, E., et al. (2016). Correction: Corrigendum: Methylation of Ribosomal RNA by NSUN5 Is a Conserved Mechanism Modulating Organismal Lifespan. Nat. Commun. 7, 11530. doi:10.1038/ncomms11530

Schumann, U., Zhang, H.-N., Sibbritt, T., Pan, A., Horvath, A., Gross, S., et al. (2020). Multiple Links between 5-methylcytosine Content of mRNA and Translation. BMC Biol. 18, 40. doi:10.1186/s12915-020-00769-5

Sharma, S., Yang, J., Watzinger, P., Kötter, P., and Entian, K.-D. (2013). Yeast Nop2 and Rcm1 Methylate C2870 and C2278 of the 25S rRNA, Respectively. Nucleic Acids Res. 41, 9062-9076. doi:10.1093/nar/gkt679

Shi, H., Wang, X., Lu, Z., Zhao, B. S., Ma, H., Hsu, P. J., et al. (2017a). YTHDF3 Facilitates Translation and Decay of N6-Methyladenosine-Modified RNA. Cel Res. 27, 315-328. doi:10.1038/cr.2017.15

Shi, M., Zhang, H., Wu, X., He, Z., Wang, L., Yin, S., et al. (2017b). ALYREF Mainly Binds to the $5^{\prime}$ and the $3^{\prime}$ Regions of the mRNA In Vivo. Nucleic Acids Res. 45, 9640-9653. doi:10.1093/nar/gkx597

Shi, H., Wei, J., and He, C. (2019). Where, when, and How: Context-dependent Functions of RNA Methylation Writers, Readers, and Erasers. Mol. Cel 74, 640-650. doi:10.1016/j.molcel.2019.04.025

Shi, Q., Xue, C., Yuan, X., He, Y., and Yu, Z. (2020a). Gene Signatures and Prognostic Values of m1A-Related Regulatory Genes in Hepatocellular Carcinoma. Sci. Rep. 10, 15083. doi:10.1038/s41598-020-72178-1

Shi, Y., Zhuang, Y., Zhang, J., Chen, M., and Wu, S. (2020b). METTL14 Inhibits Hepatocellular Carcinoma Metastasis through Regulating EGFR/PI3K/AKT Signaling Pathway in an m6A-dependent Manner. Cancer Manag. Res. 12, 13173-13184. doi:10.2147/cmar.S286275

Shimada, K., Nakamura, M., Anai, S., De Velasco, M., Tanaka, M., Tsujikawa, K., et al. (2009). A Novel Human AlkB Homologue, ALKBH8, Contributes to Human Bladder Cancer Progression. Cancer Res. 69, 3157-3164. doi:10.1158/ 0008-5472.Can-08-3530

Sun, Z., Xue, S., Zhang, M., Xu, H., Hu, X., Chen, S., et al. (2020). Aberrant NSUN2Mediated m5C Modification of H19 lncRNA Is Associated with Poor Differentiation of Hepatocellular Carcinoma. Oncogene 39, 6906-6919. doi:10.1038/s41388-020-01475-w

Teng, W., Liu, Y.-C., Jeng, W.-J., and Su, C.-W. (2021). Tertiary Prevention of HCC in Chronic Hepatitis B or C Infected Patients. Cancers 13, 1729. doi:10.3390/ cancers 13071729 
Torres, I. O., and Fujimori, D. G. (2015). Functional Coupling between Writers, Erasers and Readers of Histone and DNA Methylation. Curr. Opin. Struct. Biol. 35, 68-75. doi:10.1016/j.sbi.2015.09.007

Tourrière, H., Gallouzi, I.-e., Chebli, K., Capony, J. P., Mouaikel, J., Van Der Geer, P., et al. (2001). RasGAP-associated Endoribonuclease G3Bp: Selective RNA Degradation and Phosphorylation-dependent Localization. Mol. Cel Biol. 21, 7747-7760. doi:10.1128/mcb.21.22.7747-7760.2001

Tuorto, F., Herbst, F., Alerasool, N., Bender, S., Popp, O., Federico, G., et al. (2015). The tRNA Methyltransferase Dnmt2 Is Required for Accurate Polypeptide Synthesis during Haematopoiesis. Embo J 34, 2350-2362. doi:10.15252/ embj.201591382

Wang, X., Lu, Z., Gomez, A., Hon, G. C., Yue, Y., Han, D., et al. (2014). N6methyladenosine-dependent Regulation of Messenger RNA Stability. Nature 505, 117-120. doi:10.1038/nature12730

Wang, M., Yang, Y., Yang, J., Yang, J., and Han, S. (2020a). circ_KIAA1429 Accelerates Hepatocellular Carcinoma Advancement through the Mechanism of m6A-YTHDF3-Zeb1. Life Sci. 257, 118082. doi:10.1016/j.lfs.2020.118082

Wang, X., Li, Z.-T., Yan, Y., Lin, P., Tang, W., Hasler, D., et al. (2020b). LARP7Mediated U6 snRNA Modification Ensures Splicing Fidelity and Spermatogenesis in Mice. Mol. Cel 77, 999-1013. doi:10.1016/ j.molcel.2020.01.002

Wen, J., Lv, R., Ma, H., Shen, H., He, C., Wang, J., et al. (2018). Zc3h13 Regulates Nuclear RNA m6A Methylation and Mouse Embryonic Stem Cell Self-Renewal. Mol. Cel 69, 1028-1038. doi:10.1016/j.molcel.2018.02.015

Xue, C., Zhao, Y., and Li, L. (2020). Advances in RNA Cytosine-5 Methylation: Detection, Regulatory Mechanisms, Biological Functions and Links to Cancer. Biomark Res. 8, 43. doi:10.1186/s40364-020-00225-0

Yamashita, T., Higashi, M., Momose, S., Morozumi, M., and Tamaru, J.-I. (2017). Nuclear Expression of $\mathrm{Y}$ Box Binding-1 Is Important for Resistance to Chemotherapy Including Gemcitabine in TP53-Mutated Bladder Cancer. Int. J. Oncol. 51, 579-586. doi:10.3892/ijo.2017.4031

Yamato, I., Sho, M., Shimada, K., Hotta, K., Ueda, Y., Yasuda, S., et al. (2012). PCA1/ALKBH3 Contributes to Pancreatic Cancer by Supporting Apoptotic Resistance and Angiogenesis. Cancer Res. 72, 4829-4839. doi:10.1158/00085472.Can-12-0328

Yang, X., Yang, Y., Sun, B.-F., Chen, Y.-S., Xu, J.-W., Lai, W.-Y., et al. (2017). 5methylcytosine Promotes mRNA export - NSUN2 as the Methyltransferase and ALYREF as an m5C Reader. Cel Res. 27, 606-625. doi:10.1038/cr.2017.55

Yang, Y., Wang, L., Han, X., Yang, W.-L., Zhang, M., Ma, H.-L., et al. (2019). RNA 5-Methylcytosine Facilitates the Maternal-To-Zygotic Transition by Preventing Maternal mRNA Decay. Mol. Cel 75, 1188-1202. doi:10.1016/ j.molcel.2019.06.033
Yang, N., Wang, T., Li, Q., Han, F., Wang, Z., Zhu, R., et al. (2021). HBXIP Drives Metabolic Reprogramming in Hepatocellular Carcinoma Cells via METTL3mediated m6A Modification of HIF-1a. J. Cel Physiol. 236, 3863-3880. doi:10.1002/jcp.30128

Yin, H., Ma, X., He, Y., Liang, R., Wang, Y., Zhang, M., et al. (2020). Effect of an Outpatient Copayment Scheme on Health Outcomes of Hypertensive Adults in a Community-Managed Population in Xinjiang, China. PLoS One 15, e0238980. doi:10.1371/journal.pone.0238980

Zhang, L. N., Zhao, L., Yan, X. L., and Huang, Y. H. (2019). Loss of G3BP1 Suppresses Proliferation, Migration, and Invasion of Esophageal Cancer Cells via Wnt/ $\beta$-catenin and PI3K/AKT Signaling Pathways. J. Cel Physiol. 234, 20469-20484. doi:10.1002/jcp.28648

Zheng, G., Dahl, J. A., Niu, Y., Fedorcsak, P., Huang, C.-M., Li, C. J., et al. (2013). ALKBH5 Is a Mammalian RNA Demethylase that Impacts RNA Metabolism and Mouse Fertility. Mol. Cel 49, 18-29. doi:10.1016/j.molcel.2012.10.015

Zheng, Y. F., Zhang, X. Y., and Bu, Y. Z. (2019). LINC01133 Aggravates the Progression of Hepatocellular Carcinoma by Activating the PI3K/AKT Pathway. J. Cel Biochem. 120, 4172-4179. doi:10.1002/jcb.27704

Zhou, Y., Huan, L., Wu, Y., Bao, C., Chen, B., Wang, L., et al. (2020). LncRNA ID2-AS1 Suppresses Tumor Metastasis by Activating the HDAC8/ID2 Pathway in Hepatocellular Carcinoma. Cancer Lett. 469, 399-409. doi:10.1016/j.canlet.2019.11.007

Zhuang, J., Lin, C., and Ye, J. (2020). m 6 A RNA Methylation Regulators Contribute to Malignant Progression in Rectal Cancer. J. Cel Physiol. 235, 6300-6306. doi:10.1002/jcp.29626

Conflict of Interest: The authors declare that the research was conducted in the absence of any commercial or financial relationships that could be construed as a potential conflict of interest.

Publisher's Note: All claims expressed in this article are solely those of the authors and do not necessarily represent those of their affiliated organizations, or those of the publisher, the editors and the reviewers. Any product that may be evaluated in this article, or claim that may be made by its manufacturer, is not guaranteed or endorsed by the publisher.

Copyright (c) $2021 \mathrm{Xu}$, Zhang, Zhang, Yu, Sun, He and Guo. This is an open-access article distributed under the terms of the Creative Commons Attribution License (CC $B Y)$. The use, distribution or reproduction in other forums is permitted, provided the original author(s) and the copyright owner(s) are credited and that the original publication in this journal is cited, in accordance with accepted academic practice. No use, distribution or reproduction is permitted which does not comply with these terms. 